\title{
Chronische lymphatische Leukämie
}

\section{Therapiealltag im Fokus - Hochrisiko-Patienten mit doppelt refraktärer CLL}

Die Diagnostik und Behandlung der chronischen lymphatischen Leukämie (CLL) hat sich in den letzten Jahren deutlich verbessert: Die Fortschritte in der Therapie haben in vielen Fällen CLL-Patienten einen Überlebensvorteil verschafft. Gleichzeitig sind die Anforderungen an die Therapieentscheidungen komplexer geworden und erfordern differenziertere Herangehensweisen als noch vor einer Dekade. Wie im Rahmen der diesjährigen EHA-Jahrestagung in Amsterdam beim GSK-Satellitensymposium «Translating knowledge into practice - sharing experience in refractory CLL» deutlich wurde, sind bestimmte Patientensubgruppen nach wie vor häufig therapierefraktär, zeigen einen aggressiven Krankheitsverlauf und bedürfen verbesserter Therapieansätze.

Die CLL macht ca. 25\% aller Non-HodgkinLymphome bzw. ca. 30\% aller Leukämien bei Erwachsenen aus [1]. Die CLL wird gemäß WHO-Klassifikation als indolentes durch einen leukämischen Verlauf gekennzeichnet ist und aus der B-Zellreihe hervorgeht (Abb. 1).

Über lange Zeit nur palliativ oder mit Chlorambucil behandelt, hat sich die Prognose der CLL-Patienten durch die erweiterten therapeutischen Möglichkeiten in den letzten Dekaden Schritt für Schritt verbessert: Über eine Mono-Chemotherapie mit dem Purinanalogon Fludarabin $(\mathrm{F})$, die Kombination (lymphozytisches) Lymphom definiert, das

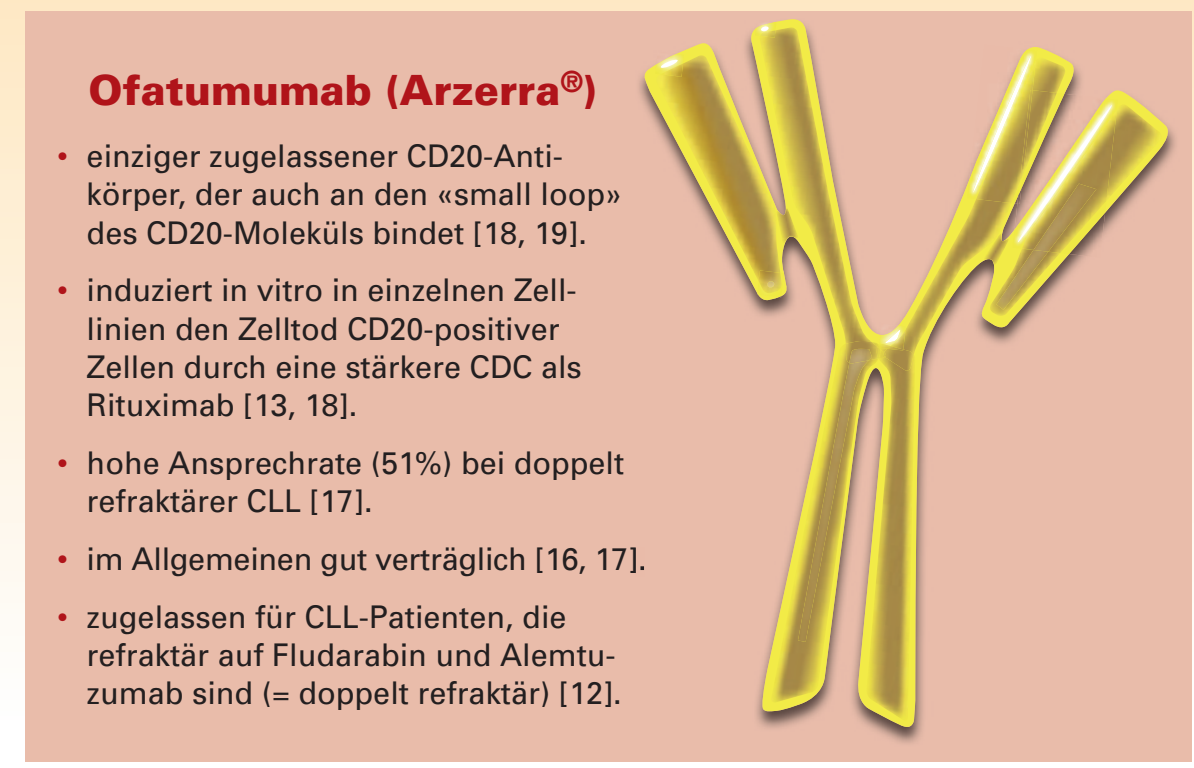

dieser Substanz mit dem Alkylanz Cyclophosphamid (C) bis hin zur Immunchemotherapie, d.h. der Addition eines monoklonalen Antikörpers wie z.B. Rituximab (R) zu dieser Therapie, ließen sich die Ansprechraten steigern, das Potenzial für eine Langzeitremission erhöhen und das Überleben verlängern [1-3].

Bei ausreichender körperlicher «Fitness», normaler Nierenfunktion und fehlenden signifikanten Komorbiditäten ist FCR daher

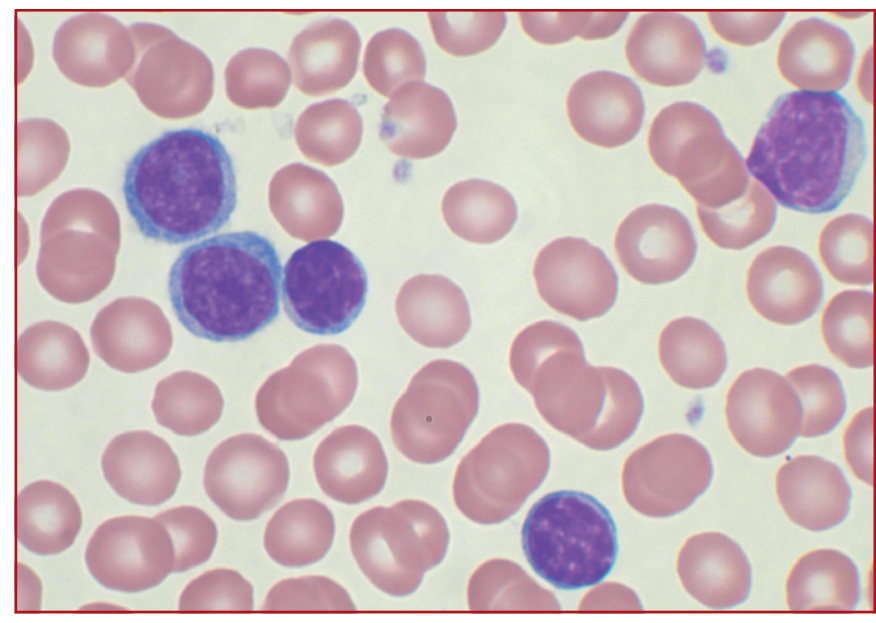

Abb. 1. Zytologische Untersuchung des Blutausstrichs: Charakteristisch sind kleine, monomorphe Lymphozyten mit schmalem Zytoplasma. heute Standardtherapie [4]. Bendamustin plus Rituximab (BR) kann eine Alternative sein, vor allem bei eingeschränkter Nierenfunktion oder Autoimmunhämolyse. Als kurative Therapieoption steht jedoch weiterhin nur die allogene Stammzelltransplantation für ausgewählte Patienten zur Verfügung.

Nach Ansicht von Dr. Francesc Bosch vom Universitätsklinikum in Barcelona, dem Leiter der spanischen CLL-Studiengruppe GELLC, bestimmt allerdings der Umgang mit «unfitten» Patienten, die an zahlreichen Begleiterkrankungen leiden, den Behandlungsalltag. So beträgt bereits heute das mediane Alter der CLL-Patienten bei Erstdiagnose 70 Jahre bei Männern bzw. 72 Jahre bei Frauen [4]. Mit dem Alter nehmen jedoch auch die Komorbiditäten der Patienten $\mathrm{zu}$, bei gleichzeitig verkürztem Gesamtüberleben und reduzierter möglicher Therapieintensivität $[5,6]$.

\section{Herausforderungen an das moderne CLL-Management}

Als prognostisch relevant für die CLL wurden bereits mehrere biologische Faktoren ermittelt, darunter die Deletion von 17p13 und die zumeist damit einhergehende TP53Mutation: Diese Risikofaktoren, die in ca. 
$10 \%$ aller Fälle bereits bei Erstdiagnose und bei immerhin ca. $20 \%$ aller Patienten im Rezidiv vorliegen, weisen auf eine ungünstige Prognose und ein hohes Risiko für Therapieresistenz hin [7]. Auch die CLLLeitlinie der DGHO empfiehlt daher, das Vorliegen einer 17p13-Deletion diagnostisch zu prüfen und bei der Therapiewahl zu berücksichtigen [4].

Darüber hinaus wurden kürzlich bei etwa $10 \%$ der CLL-Patienten sogenannte NOTCH1-Mutationen beobachtet, die möglicherweise ebenfalls mit einer ungünstigeren Überlebensprognose assoziiert sind, berichtete Bosch [8, 9].

Die Frage, inwieweit die neueren zyto- oder molekulargenetischen Befunde auch bei differenzialtherapeutischen Überlegungen auBerhalb von klinischen Studien eine Rolle spielen könnten, sei aber derzeit noch nicht abschließend geklärt. Bosch zufolge befindet sich die Therapieplanung aktuell in einem Spannungsfeld zwischen Maximierung der Effektivität und Minimierung der Toxizität. Bei der oftmals betagten Patientenpopulation verbieten sich dabei nicht selten intensive Therapieverfahren wie eine Stammzelltransplantation. Der derzeit vielleicht wichtigste Ansatz zur Therapieoptimierung besteht Bosch zufolge darin, Patientensubgruppen zu selektieren, die von bestimmten Therapieoptionen profitieren.

\section{Refraktäre CLL-Patienten benöti- gen alternative Therapieoptionen}

Wie Dr. Ann Janssens vom Universitätsklinikum zu Leuven erklärte, spricht man gemäß der Leitlinie der Internationalen CLL-Arbeitsgruppe (IWCLL) bei Krankheitsprogredienz einer CLL später als 6 Monate nach Therapieende von einem Rezidiv. Als refraktär hingegen gilt die Erkrankung bei primärem Therapieversagen oder bei Progression innerhalb eines Zeitrahmens von 6 Monaten nach Therapieende [10].

Für die therapierefraktäre Situation würden Standardtherapien jedoch fehlen, betonte Janssens: Nach wie vor haben CLL-Patienten, die sich gegenüber den derzeitigen Standardtherapien wie FCR oder BR als refraktär erweisen oder nur eine kurze Remission von weniger als 2 Jahren Dauer erzielen, eine ungünstige Prognose: Ihre mittlere Gesamtüberlebenszeit beträgt nur 1-2 Jahre ab dem Zeitpunkt der Salvage-Therapie [4].

Daher, so Janssens, würde auch von der «Ultra High Risk»-CLL gesprochen, der neben Patienten mit biologischen Markern

\section{CLL-Kasuistik: 61-jähriger doppelt refraktärer Patient}

- CLL-Erstdiagnose erfolgte 2006, als der Patient mit einer Lymphadenopathie vorstellig wurde

\section{- Begleiterkrankung: Hypertonus}

- Frühere Therapiemaßnahmen: - Chlorambucil

(2006, 8 Zyklen, partielle

Remission)

- R-CVP (Rituximab + Cyclophosphamid/Vincristin/Prednisolon) (2007, 8 Zyklen, partielle Remission)

- FCR (Fludarabin/Cyclophosphamid/Rituximab) (2010, 6 Zyklen, zunächst partielles Ansprechen, dann aber Progress innerhalb von 6 Monaten $\rightarrow$ refraktär)

Im April 2011 weist der Patient eine Neutropenie auf $(700 / \mu \mathrm{l})$, sein $\mathrm{Hb}$ Wert liegt bei $14,3 \mathrm{~g} / \mathrm{dl}$, die Thrombozytenzahl bei $110.000 / \mu \mathrm{l}$, Leuko-

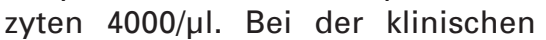
Untersuchung werden u.a. eine Lymphadenopathie (LK-Durchmesser bis zu $10 \mathrm{~cm}$ ), eine Hepatosplenomegalie sowie ein makulopapulöser Hautausschlag festgestellt. Zytogenetisch findet sich eine Deletion 13q, jedoch keine Deletion $17 p$.

In den folgenden 3 Monaten verschlechtert sich das Blutbild des Patienten (Hb: 12,6 g/dl, Thrombo-

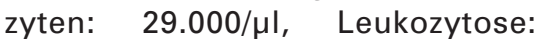

$74.000 / \mu \mathrm{l})$. Molekulargenetisch findet sich eine TP53-Mutation.

- Alemtuzumab / Dexamethason (07-11/2011, partielle Remission)

Nebenwirkungen im Therapieverlauf waren u. a. Zytopenie, bakterielle Pneumonie und CMV-Infektion.

Im Januar 2012 kommt es erneut zum therapiebedürftigen Progress, mit Blutbildverschlechterung und Fatigue.

\section{Therapiestart mit Ofatumumab (Arzerra ${ }^{\circledR}$ ) im Januar 2012}

Zwischenergebnis: Bei einer Untersuchung, die nach der 10. Ofatumumab-Infusion erfolgte*, zeigte sich die CLL des Patienten hinsichtlich Blutbild, Lymphadenopathie und Hepatosplenomegalie stabilisiert. Die Therapie hatte sich bis dato als gut verträglich erwiesen, überdies berichtete der Patient eine Verbesserung der Fatigue und seines Allgemeinzustands.

\begin{abstract}
*Das Infusionsschema beinhaltet 8 aufeinanderfolgende Ofatumumab-Infusionen im wöchentlichen Abstand, 4 bis 5 Wochen später gefolgt von 4 weiteren Infusionen in monatlichen Abständen. Die empfohlene Ofatumumab-Dosis beträgt $300 \mathrm{mg}$ für die erste Infusion und 2000 mg für alle nachfolgenden Infusionen [12].
\end{abstract}

wie 17p-Deletion oder TP53-Mutation auch Patienten mit Frührezidiv innerhalb von 24-36 Monaten nach Immunchemotherapie wie z.B. FCR- sowie Fludarabin-refraktäre Patienten zuzuordnen sind [7].

\section{Schwieriger Therapieverlauf eines 61-jährigen Patienten}

Wie Janssens und Prof. Andrew Pettitt, Royal Liverpool Universitätsklinikum, gemeinsam mit einer Patientenkasuistik demonstrieren konnten, stellt die refraktäre CLL komplexe Anforderungen an das wei- tere Therapiemanagement: Sie stellten hierzu den Fall eines 61-jährigen CLL-Patienten vor, der zunächst gut auf verschiedene (Immun-)Chemotherapien ansprach, im weiteren Krankheitsverlauf jedoch sowohl auf Fludarabin als auch auf Alemtuzumab refraktär war (s. Kasten). Dieser doppelt refraktäre Hochrisiko-Patient konnte, so Janssens und Pettitt, von einer Infusionstherapie mit Ofatumumab, die gut vertragen wurde, profitieren und bei einer aktuellen Krankheitsbeurteilung nach 10 Ofatumumab-Gaben eine Stabilisierung der Erkrankung erreichen. 


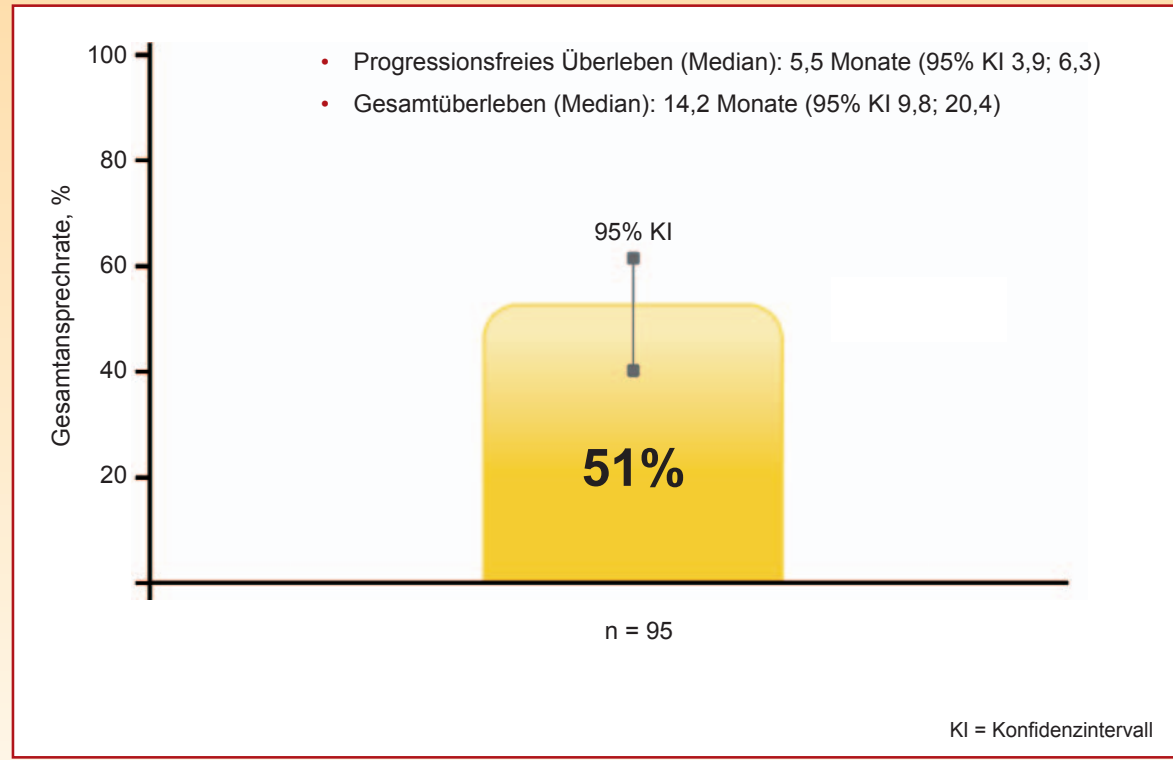

Abb. 2. Ofatumumab-Zulassungsstudie bei doppelt (Fludarabin- und Alemtuzumab-)refraktären CLL-Patienten: Gesamtansprechen, beurteilt durch ein unabhängiges «Review Committee» (IRC) [17].

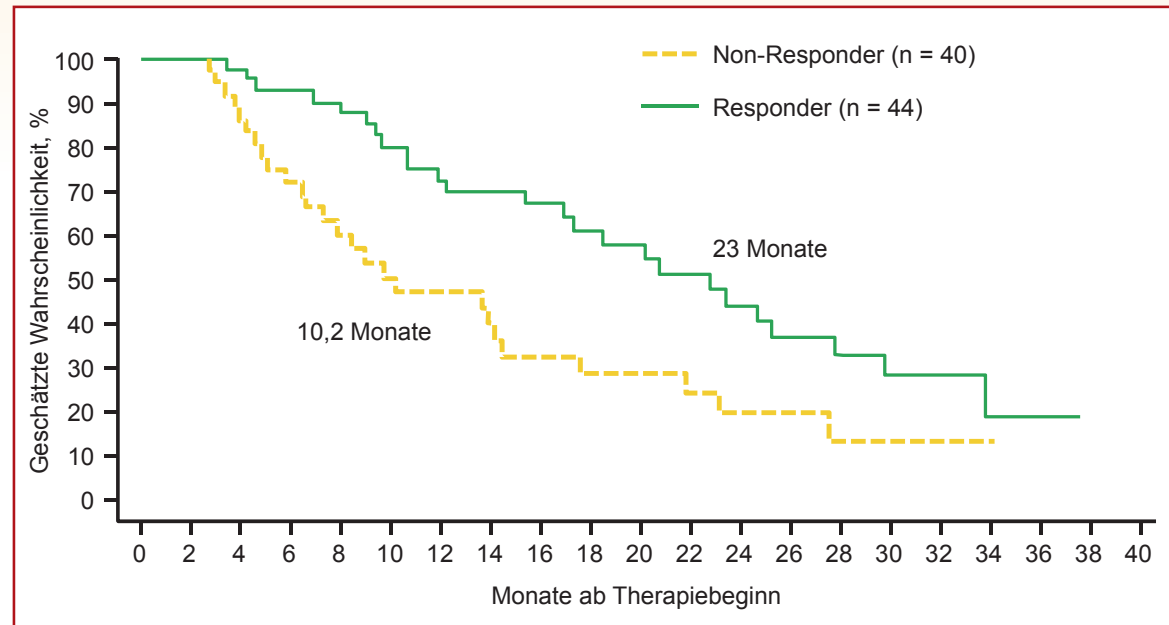

Post-hoc-Landmark-Analyse in Woche $12^{*}$

*Die Analyse beinhaltete nur die Fludarabin- und Alemtuzumabrefraktären Patienten, die in Woche 12 noch am Leben waren.

Abb. 3. Ofatumumab-Zulassungsstudie: Gesamtüberleben nach Ansprechverhalten (Median) [17].

Parallel zu diesem Fall illustrierten die beiden Referenten anhand einer retrospektiven Studie des MD Anderson Cancer Centers, bei der die Effektivität unterschiedlicher Salvage-Therapien ausgewertet wurde, wie ungünstig die Ausgangssituation bei Therapierefraktärität ist: Doppelt (Fludarabin- und Alemtuzumab-)refraktäre Patienten wiesen in diesen historischen Daten lediglich eine Ansprechrate von 20\% auf [11]. Die Ansprechraten bei Monotherapie mit monoklonalen Antikörpern (z.B. Rituximab) oder bei reinen Chemotherapie-Kombinationsregimen betrugen $0 \%$. Nur mit kombinierten Chemo-Immuntherapien konnte ein Anspre- chen erreicht werden. Die mediane Zeit bis zum Therapieversagen lag über alle SalvageTherapieregime hinweg bei 2-3 Monaten, das mediane Gesamtüberleben bei 8 Monaten. Gleichzeitig war die Rate schwerwiegender Infektionen mit $60 \%$ ausgesprochen hoch [11].

\section{Ofatumumab: Präklinische Daten}

Ofatumumab ist ein vollständig humaner monoklonaler Anti-CD20-Antikörper der zweiten Generation, der sich im Unterschied zu herkömmlichen Anti-CD20-Antikörpern gegen ein anderes therapeutisches Zielareal richtet
[13]: Während Rituximab nur am «large loop» des CD20-Moleküls bindet, ist Ofatumumab der erste zugelassene Anti-CD20-Antikörper, der zusätzlich zur großen auch an der kleinen Schleife («small loop») bindet.

Von der Bindung an die beiden extrazellulären Schleifen des CD20-Moleküls wird eine engere Antikörperbindung an die BZellmembran angenommen [14]. Die Antikörperbindung an den «small loop» scheint dabei in vitro auch die Komplement-abhängige Zytotoxizität (CDC) zu verstärken: Präklinisch zeigte sich Ofatumumab auch in Zelllinien von niedriger CD20-Dichte mit einer stärkeren CDC versus Rituximab assoziiert $[13,15]$.

\section{Ofatumumab: Zulassung für doppelt refraktäre Patienten}

Der monoklonale Anti-CD20-Antikörper ist seit April 2010 für Patienten mit CLL zugelassen, die refraktär auf Fludarabin und Alemtuzumab sind (= doppelt refraktär), d.h. die nicht oder nur weniger als 6 Monate auf Fludarabin und Alemtuzumab angesprochen hatten.

In die offene, einarmige Zulassungsstudie waren 95 doppelt refraktäre, intensiv vorbehandelte Patienten eingeschlossen: die Patienten hatten im Median bereits 5 Vortherapien erhalten, einschließlich Rituximab (59\%). Das mediane Alter der Patienten lag bei 64 Jahren, wobei sich die meisten Patienten im Binet-Stadium C bzw. im Rai-Stadium III/IV befanden (etwa 60\%) [16, 17].

Primärer Studienendpunkt war die objektive Ansprechrate, während die sekundären Endpunkte die Ansprechdauer, das progressionsfreie Überleben, das Gesamtüberleben sowie die Sicherheit der Therapie umfassten.

Nach einer Zusammenfassung von Janssens sind folgende Ergebnisse der OfatumumabZulassungsstudie besonders praxisrelevant (Abb. 2-4) [17]:

- Die Gesamtansprechrate der Mono-Immuntherapie mit Ofatumumab betrug $51 \%$, was einem deutlich höheren Anteil entsprach, als retrospektive historische Daten zu Salvage-(Immun)-Chemotherapien bei diesem doppelt refraktären Patientenkollektiv zeigten (20\%).

Mit Ofatumumab konnte auch in Hochrisikogruppen ein Ansprechen erzielt werden (44\% Ansprechrate bei FCR-refraktären Patienten, 37\% Ansprechrate bei Patienten mit 17p-Deletion). 


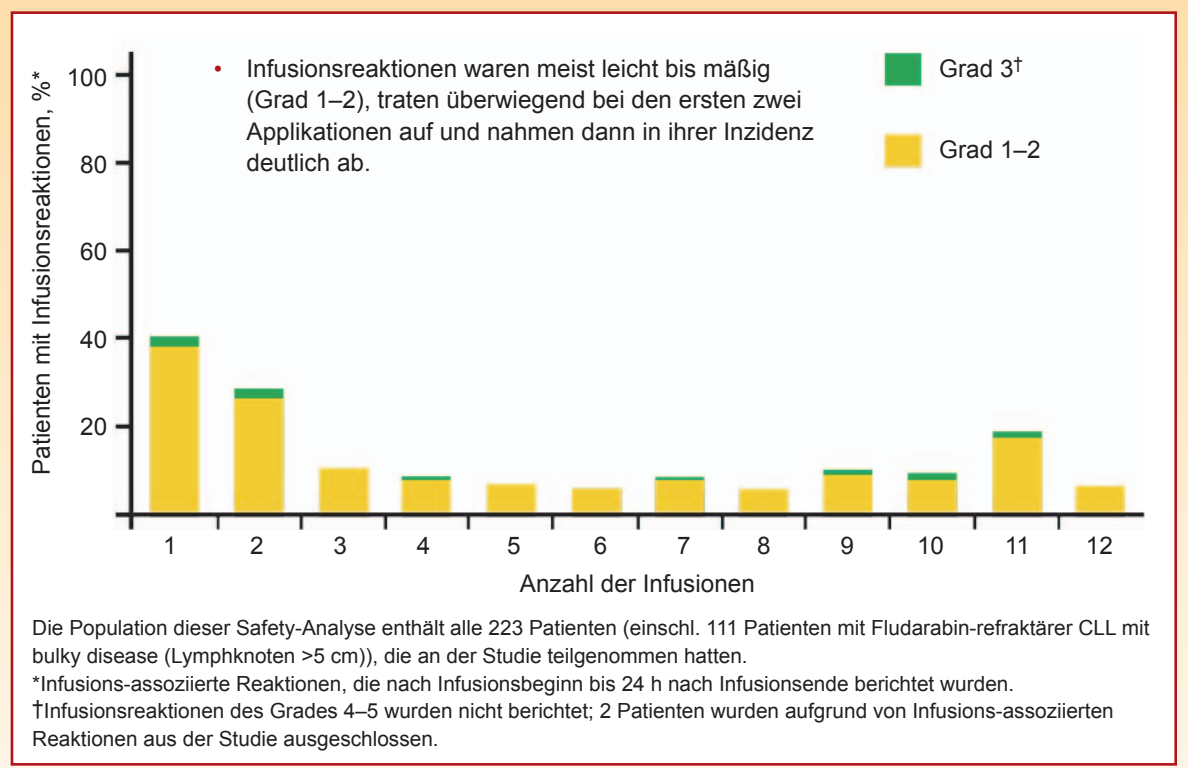

Abb. 4. Ofatumumab-Zulassungsstudie: Infusionsassoziierte Reaktionen [17].

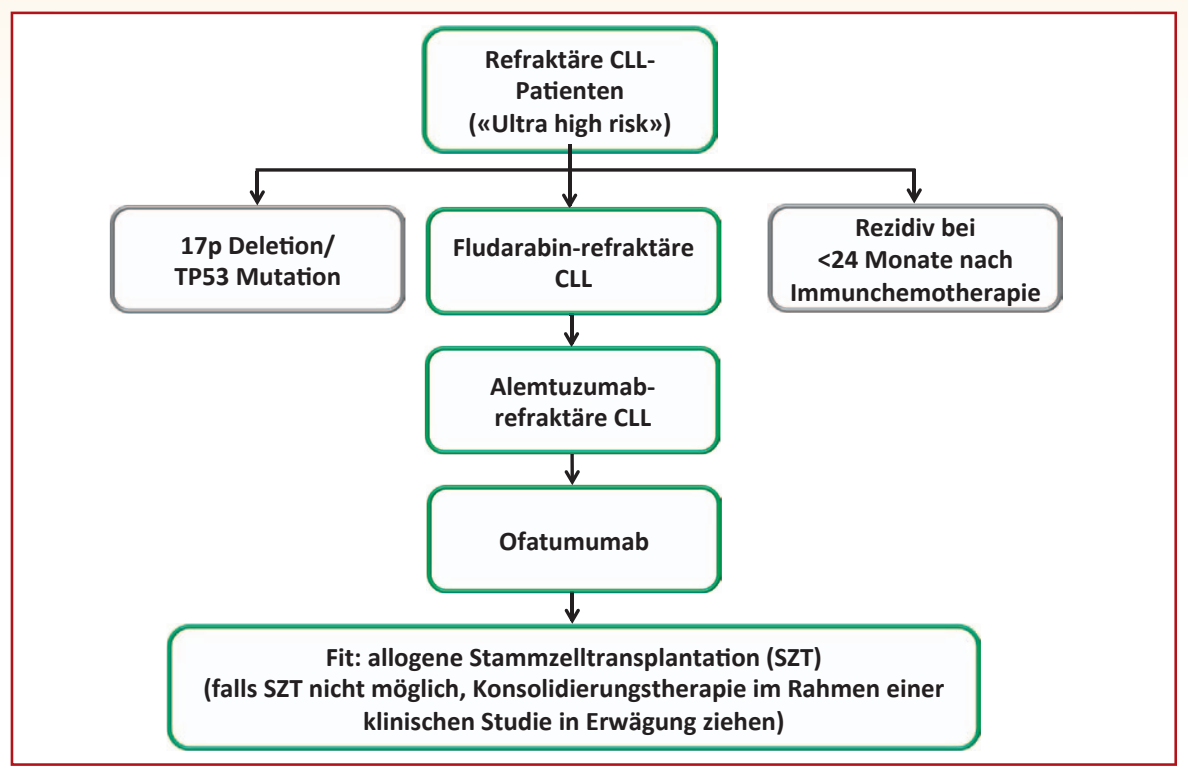

Abb. 5. Vorschlag für einen Therapiealgorithmus mit Ofatumumab (nach einem Vortrag von Dr. Ann Janssens).

- Das mediane progressionsfreie Überleben $(95 \% \mathrm{KI})$ lag bei $5,5(3,9-6,3)$ Monaten, das mediane Gesamtüberleben bei 14,2 (9,8-20,4) Monaten.

- Patienten, die auf die Behandlung mit Ofatumumab ansprachen, hatten ein im Median um ca. 1 Jahr verlängertes Gesamtüberleben (entsprechend LandmarkAnalyse in Woche 12).

- Die Behandlung mit Ofatumumab wurde im Allgemeinen gut vertragen. Die Mehrzahl der unerwünschten Ereignisse entsprach Grad 1 und 2.

Häufigste Nebenwirkungen vom Grad $\geq 3$ waren Pneumonien (10\%) und Neutropenie $(13 \%)$, was bei diesem Patientenkollektiv in etwa zu erwarten ist. Infusionsreaktionen traten meist bei der 1 . und 2. Infusion auf und weniger häufig während der nachfolgenden Infusionen.

- Ofatumumab ist den Studienergebnissen zufolge nicht kontraindiziert bei myelosupprimierten Patienten.

- Auch Rituximab-vorbehandelte Patienten sprachen auf die Behandlung mit Ofatumumab mit einer ähnlichen Häufigkeit an wie Patienten, die zuvor kein Rituximab erhalten hatten.
Möglicher Therapiealgorithmus mit Ofatumumab in der Praxis

Aus Janssens Perspektive erfüllt Ofatumumab die Voraussetzungen für eine relevante Therapieoption bei doppelt refraktärer Hochrisiko-CLL, um eine Stabilisierung des Patienten $\mathrm{zu}$ ermöglichen und ggf. die Option einer kurativen allogenen Stammzelltransplantation realisierbar $\mathrm{zu}$ machen (Abb. 5).

Dr. Yuri Sankawa, Stuttgart

\section{Referenzen}

1 Hallek M, Pflug N: Ann Oncol 2010;21 (suppl 7): vii154-vii164.

2 Hallek M, et al.: Hematology Am Soc Hematol Educ Program 2009;440-449.

3 Sellner L, et al.: Curr Hematol Malig Rep 2012; 7:3-12.

4 Wendtner CM, et al.: DGHO-Leitlinie CLL. Stand: Januar 2011.

5 Yancik R: Cancer 1997;80:1273-1283.

6 Kuderer NM, et al.: Cancer 2006;106:2258-2266.

7 Stilgenbauer S, Zenz T: Am Soc Hematol Educ Program 2010;481-488.

8 Puente XS, et al.: Nature 2011;475:101-105.

9 Fabbri G, et al.: J Exp Med 2011;208:1389-1401.

10 Hallek M et al.: Blood 2008;111:5446-5456.

11 Tam CS, et al.: Leuk Lymphoma 2007;48:1931-1939.

12 Fachinformation Arzerra ${ }^{\circledR}$.

13 Teeling JL, et al.: Blood 2004,104:1793-1800

14 Nightingale G: Ann Pharmacother 2011;45:1248-1255.

15 Österborg A: Expert Opin Biol Ther 2010;10:439-449.

16 Wierda WG, et al.: J Clin Oncol 2010,28:1749-1755.

17 Wierda WG, et al.: Blood 2010;116:406 (abstr 912 und oral presentation).

18 Teeling JL, et al.: J Immunol 2006;177:362-371.

19 Pawluczkowycz AW, et al.: J Immunol 2009;183: 749-758.

\section{Quelle}

GSK-Satellitensymposium «Translating knowledge into practice - sharing experience in refractory CLL», im Rahmen des 17. EHA-Kongresses, 14. Juni 2012 , Amsterdam.

\begin{tabular}{l} 
Impressum \\
\hline Chronische Iymphatische Leukämie \\
Therapiealltag im Fokus - \\
Hochrisiko-Patienten mit doppelt \\
refraktärer CLL \\
PharmaForum in Onkologie 35 I 9 I 12 \\
@ 2012 by S. Karger Verlag für Medizin \\
und Naturwissenschaften GmbH \\
Wilhelmstraße 20A \\
79098 Freiburg, Deutschland \\
\hline Mit freundlicher Unterstützung durch \\
GlaxoSmithKline GmbH \& Co. KG. \\
Verlag, Herausgeber, Redaktion und Verlagsgeschäftsführung \\
übernehmen keine Verantwortung für den Inhalt dieser Rubrik.
\end{tabular}

